\title{
Gout and dementia in the elderly: a cohort study of Medicare claims
}

\author{
Jasvinder A. Singh ${ }^{1,2,3,4^{*}}$ (D) and John D. Cleveland ${ }^{2,4}$
}

\begin{abstract}
Background: Conflicting data in the literature raise the question whether gout, independent of its treatment, increases the risk of dementia in the elderly. Our objective was to assess whether gout in older adults is associated with the risk of incident dementia.

Methods: We used the 5\% Medicare claims data for this observational cohort study. We used multivariable-adjusted Cox proportional hazard models to assess the association of gout with a new diagnosis of dementia (incident dementia), adjusting for potential confounders/covariates including demographics (age, race, sex), comorbidities (Charlson-Romano comorbidity index), and medications commonly used for cardiac diseases (statins, beta-blockers, diuretics, and angiotensin converting enzyme (ACE)-inhibitors) and gout (allopurinol and febuxostat).
\end{abstract}

Results: In our cohort of 1.71 million Medicare beneficiaries, 111,656 had incident dementia. The crude incidence rates of dementia in people without and with gout were 10.9 and 17.9 per 1000 person-years, respectively. In multivariable-adjusted analyses, gout was independently associated with a significantly higher hazard ratio of incident dementia, with a HR of $1.15(95 \% \mathrm{Cl}, 1.12,1.18)$; sensitivity analyses confirmed the main findings. Compared to age 65 to $<75$ years, age 75 to $<85$ and $\geq 85$ years were associated with 3.5 and 7.8 -fold higher hazards of dementia; hazards were also higher for females, black race or people with higher medical comorbidity.

Conclusion: Gout was independently associated with a 15\% higher risk of incident dementia in the elderly. Future studies need to understand the pathogenic pathways involved in this increased risk.

Keywords: Gout, Dementia, Risk, Older adults, Medicare, Claims database

\section{Background}

Gout, the most common inflammatory arthritis in the adults, is characterized by hyperuricemia, monosodium urate crystal formation and inflammation. Dementia, characterized by progressive deterioration of cognitive ability and function, is a common disease of the elderly that has replaced ischemic heart disease as the leading cause of death in England and Wales [1].

Dementia can be caused by Alzheimer's disease (60$70 \%)$, vascular dementia (20\%), and other conditions such as Parkinson's disease, etc. [2]. Dementia is frequently diagnosed based on the clinical presentation. Various clinical tests used for the evaluation of cognitive

\footnotetext{
* Correspondence: Jasvinder.md@gmail.com

${ }^{1}$ Medicine Service, Birmingham VA Medical Center 700 19th St S,

Birmingham, AL 35233, USA

${ }^{2}$ Department of Medicine at School of Medicine, University of Alabama at Birmingham, 1720 Second Ave. South, Birmingham, AL 35294-0022, USA
} Full list of author information is available at the end of the article function i.e. mini mental state examination (MMSE) [3], global deterioration scale (GDS) [4] etc., can document the severity of cognitive impairment quantitatively and allow the assessment of cognitive changes over time [5]. Diagnostic criteria for various dementia subtypes have been developed, including the NINDS-AIREN criteria [6], NINCDS-ADRDA criteria [7], etc. Additionally, the recognition of biomarkers for certain subtypes, such as Alzheimer's disease including structural MRI, molecular neuroimaging with PET, and cerebrospinal fluid analyses are bridging the knowledge gap between pathophysiology and clinical manifestations $[8,9]$.

Dementia is associated with limitation of functional ability [10] and deficits in quality of life [11], which can lead to the loss of independence and increased morbidity and mortality $[12,13]$. The number of people with dementia worldwide is expected to quadruple to 115 million by 2050 [14]. Therefore, dementia is a significant public 
health problem of increasing impact. Studies are needed to identify novel risk factors for dementia beyond demographics, cardiovascular disease and head injury [15]. Identification of novel risk factors can lead to back-to-the-bench translational studies and identify new pathways and mechanisms of dementia.

The pursuit of a link between gout/hyperuricemia and dementia has led to contradictory results. Most observational studies, including population-based studies, showed that hyperuricemia was associated with a higher risk of dementia and cognitive dysfunction [16-21], while a few studies found hyperuricemia to be associated with a lower risk of dementia [22, 23].

Recently, a large French population-based study in the elderly (65 years or older) showed that hyperuricemia was associated with a higher risk of dementia and with MRI changes of aging in the brain (extensive white matter hyperintensity volume; $p=0.10$ ), providing the first clinical-pathological correlation to-date between hyperuricemia and brain changes [24]. One of the studies that found gout to be associated with a reduced risk of dementia found this risk reduction to be limited to treated gout patients (urate-lowering drugs or colchicine) and no association was found in untreated gout patients [23], indicating that protection against dementia risk in patients with gout may be medication-related. These observations raise the question whether gout, independent of its treatment, increases the risk of dementia in the elderly, which our study aimed to address. As a secondary study objective, we also assessed whether this risk varied by demographic features or the presence of common comorbidities.

\section{Methods}

\section{Data sources and study sample}

We used the 5\% random Medicare claims sample from 2006 to 2012 for this cohort study. Data were obtained from the Centers for Medicare and Medicaid Services (CMS) data warehouse. We followed the with the Strengthening of Reporting in Observational studies in Epidemiology (STROBE) guideline for reporting the methods and results of our study. People were eligible for this study if they were enrolled in Medicare fee-for-service (Parts A, B) and not enrolled in a Medicare Advantage Plan from 2006 to 2012, and resided in the U.S. The study was approved by the Institutional Review Board (IRB) at the University of Alabama at Birmingham (UAB).

\section{Predictor of interest and covariates}

Gout, confirmed by the existence of two claims at least 4-weeks apart with an International Classification of Diseases, ninth revision, common modification (ICD-9-CM) code of 274.xx, was our main predictor of interest. This definition has high accuracy with sensitivity of $90 \%$ and specificity of $100 \%$ [25]. People without gout at baseline were are the risk of being diagnosed with gout during the follow-up. The diagnosis of gout had to precede the diagnosis of dementia, i.e., we included all prevalent cases of gout at the beginning of study window and all new gout cases during study period, as long as the gout diagnosis preceded the diagnosis of dementia. We excluded people with only one claim for gout from the analysis.

We included several covariates and potential confounders. Demographic characteristics including age, sex and race were obtained from the Medicare beneficiary summary file. Comorbidity was assessed from all inpatient and outpatient claim files that included diagnosis codes and claim dates from Medicare part $A$ and $B$ files, and calculated using the Charlson-Romano index, which is a commonly used validated weighted comorbidity index, developed for the claims data [26]. Similarly, we used ICD-9-CM codes from all inpatient and outpatient claim files to identify hypertension, hyperlipidemia, and coronary artery disease. All demographics except age were assessed at baseline. Age was a time-varying covariate, which was allowed to change during the study follow-up. Common medications for the treatment of cardiovascular diseases (statins, beta-blockers, diuretics, and angiotensin converting enzyme (ACE)-inhibitors) and for urate-lowering in gout (allopurinol and febuxostat) were included, data derived from the Medicare part D file that contains all prescription claims (dose, supply, and drug name). Medication use variables were modeled as time-varying covariates, i.e., allowed to vary throughout the study period.

\section{Dependent variable/outcome of interest}

The outcome of interest was incident dementia, identified by the occurrence of two claims at least 4-weeks apart with ICD-9-CM codes for 290.xx, 294.1x, or 331.2, using the same codes as the Quan-Charlson index, a validated medical comorbidity index, with no claims for dementia in the baseline period of at least 1 year $(1 / 1 /$ 2005 to $12 / 31 / 2005)$. This approach is valid, with positive and negative predictive values of 96 and $98 \%$ and specificity of $100 \%$ [27].

\section{Statistical analyses}

We used a person-day file, assessing risk of outcome each day of follow-up. The time origin for analyses was the time for the second claim of gout. The study follow-up for each person was censored at the time of the occurrence of incident dementia, end of the study period $(12 / 31 / 2012)$ or death, whichever occurred first. 
Crude incidence rates were calculated per 1000 person-years for incident dementia in people with or without gout. Unadjusted characteristics were compared using a t-test or a chi-square test, as appropriate. We used a multivariable-adjusted Cox proportional hazard analysis to examine the association of gout with incident dementia, controlling for demographics, comorbidity and medication use (model 1). We calculated hazard ratios (HR) and 95\% confidence intervals (CI). Sensitivity analyses substituted continuous Charlson-Romano index score with categorical score (model 2) or individual comorbidities plus hypertension, hyperlipidemia, and coronary artery disease (model 3).

\section{Results}

There were 1,712,821 Medicare beneficiaries in our study cohort. Of these, 111,656 had incident dementia during the study follow-up. With 106,346 incident dementia cases in people without gout and 5310 dementia cases in people with gout, the crude incidence rates were 10.9 and 17.9 per 1000 person-years, respectively. The mean (SD) [median; interquartile range] time from the diagnosis of gout to the diagnosis of dementia was 2.3 years (1.7) [2.0; 0.9 to 3.5$]$.

Compared to people who did not develop dementia, people who developed incident dementia were 5 years older, more likely to be female, Black, have higher Charlson-Romano score and higher prevalence of all Charlson-Romano comorbidities except AIDS (Table 1). Hypertension, hyperlipidemia and coronary artery disease were more frequent in people with dementia, compared to people without (Table 1).

In multivariable-adjusted analyses, gout was independently associated with a significantly higher hazard ratio of incident dementia, with a HR of 1.15 (95\% CI, 1.12, 1.18), an association that persisted in sensitivity analyses (Table 2). Compared to age 65 to $<75$ years, older age groups were associated with 3.5 and 7.8-fold higher hazards of dementia; hazards were also higher for females, Black race or people with higher medical comorbidity (Table 2).

Subgroup analyses indicated that there were minor differences by race, age and sex (Fig. 1), but all hazard ratios between gout and incident dementia were significant across categories of these variables. Gout was significantly associated with dementia in patients without key comorbidities (CAD, hyperlipidemia, CVD, diabetes, hypertension) with HR ranging $1.2-1.5$, but not in patients with each of these comorbidities, except a borderline association in those with CAD, with HR of 1.07 (95\% CI, 1.01 to 1.14; Fig. 1).

\section{Discussion}

In this study, we found that elderly people with gout had a $15-18 \%$ higher hazard of dementia, compared to people without gout, after adjusting for effects of age, sex, race, medical comorbidities, and common medications for cardiac diseases and gout. As expected this association was statistically significant in this study with a large sample size, but the relative increase in risk was small. On the other hand, the population of Americans 65 years or older is predicted to grow from 34.4 million in 2000 to $>70$ million in 2030 [28]. Given the significant associated morbidity and mortality of dementia in this age group and its common occurrence in this age group [1,29-31], even this small increase in dementia risk makes this finding clinically relevant. Older age was a strong risk factor for dementia, although female sex, Black race and higher comorbidity were also significantly associated with higher risk of dementia.

A higher risk of dementia in people with gout should not be surprising, since gout is associated with hyperuricemia, chronic inflammation and oxidative stress, and several or all of these mechanisms may play a key role in the pathogenesis of dementia. Recently noted positive association of hyperuricemia with MRI lesions, extensive white matter hyperintensity volume (a biomarker of small vessel disease; $p=0.10$ ) in elderly people with gout [24], is consistent with our finding of higher risk of dementia in the elderly with gout. Evidence links oxidative stress to neurodegenerative processes in dementia is accumulating [32]. Animal studies show that associated oxidative damage in brain precedes Aß-amyloid deposition and neuronal injury [33]. The conversion of hypoxanthine into uric acid by xanthine oxido-reductase (XOR) system involves two steps and leads to the formation of superoxide species that increases oxidative stress. Thus, oxidative stress may be the final common pathway in both gout and dementia, that can at least partially explain the increased risk of dementia in elderly people with gout. A better understanding of pathways that lead to higher risk of dementia in people with gout will not only improve our understanding of this association between two common conditions in the elderly population, but may also pave the path for discovery of new treatments targeting these pathways to prevent or delay the onset of dementia. The elderly population in the U.S. that is 65 years or older is predicted to grow from 34.4 million in 2000 to $>70$ million in 2030, indicating that studies of potential risk factors of common public health problems in this population, such as dementia, are needed.

Additionally, research has shown that uric acid has both a pro-oxidant action [34, 35] and an anti-oxidant action [36, 37]. While the former is potentially neurotoxic, the latter may make uric acid potentially neuroprotective. Some have proposed that hyperuricemia is associated with elevated total serum antioxidant capacity among individuals with atherosclerosis, indicating that 
Table 1 Demographic and clinical characteristics of beneficiaries who developed incident dementia ${ }^{a}$

\begin{tabular}{|c|c|c|c|c|}
\hline & \multirow[t]{2}{*}{ All beneficiaries } & \multicolumn{2}{|c|}{ Incident Dementia ${ }^{a}$ during the follow-up } & \multirow[t]{2}{*}{$p$-value } \\
\hline & & $\overline{\mathrm{No}}$ & Yes & \\
\hline Total, N (beneficiaries) & $1,712,821$ & $1,601,165$ & $111,656^{\mathrm{b}}$ & $\mathrm{N} / \mathrm{A}$ \\
\hline Age, mean (SD) & $75.2(7.5)$ & $74.9(7.5)$ & $80.0(7.1)$ & $<0.0001$ \\
\hline Sex, N (\%) & & & & $<0.0001$ \\
\hline Male & $729,781(42.6 \%)$ & $693,059(43.3 \%)$ & $36,722(32.9 \%)$ & \\
\hline Female & $983,040(57.4 \%)$ & $908,106(56.7 \%)$ & $74,934(67.1 \%)$ & \\
\hline Race/Ethnicity, N (\%) & & & & $<0.0001$ \\
\hline White & $1,476,044(86.2 \%)$ & $1,380,894(86.2 \%)$ & $95,150(85.2 \%)$ & \\
\hline Black & $139,833(8.2 \%)$ & $128,824(8.0 \%)$ & $11,009(9.9 \%)$ & \\
\hline Other/unknown & $96,944(5.7 \%)$ & $91,447(5.7 \%)$ & 5497 (4.9\%) & \\
\hline Charlson-Romano score, mean (SD) & $1.56(2.36)$ & $1.52(2.34)$ & $2.18(2.49)$ & $<0.0001$ \\
\hline Charlson-Romano score & & & & $<0.0001$ \\
\hline 0 & $912,029(53.2 \%)$ & $870,890(54.4 \%)$ & $41,139(36.8 \%)$ & \\
\hline 1 & $174,091(10.2 \%)$ & $159,811(10.0 \%)$ & $14,280(12.8 \%)$ & \\
\hline$\geq 2$ & $626,701(36.6 \%)$ & $570,464(35.6 \%)$ & $56,237(50.4 \%)$ & \\
\hline \multicolumn{5}{|l|}{ Charlson-Romano comorbidities } \\
\hline Myocardial Infarction & $67,609(3.9 \%)$ & $62,029(3.9 \%)$ & $5580(5.0 \%)$ & $<0.0001$ \\
\hline Heart Failure & $198,607(11.6 \%)$ & 180,109 (11.2\%) & $18,498(16.6 \%)$ & $<0.0001$ \\
\hline Peripheral vascular disease & $164,078(9.6 \%)$ & 147,448 (9.2\%) & $16,630(14.9 \%)$ & $<0.0001$ \\
\hline Cerebrovascular disease & $162,203(9.5 \%)$ & 142,965 (8.9\%) & $19,238(17.2 \%)$ & $<0.0001$ \\
\hline Dementia & $58,582(3.4 \%)$ & $47,204(2.9 \%)$ & $11,378(10.2 \%)$ & $<0.0001$ \\
\hline Chronic pulmonary disease & $266,474(15.6 \%)$ & 245,787 (15.4\%) & $20,687(18.5 \%)$ & $<0.0001$ \\
\hline Connective tissue disease & $47,610(2.8 \%)$ & $43,566(2.7 \%)$ & $4044(3.6 \%)$ & $<0.0001$ \\
\hline Peptic ulcer disease & $32,015(1.9 \%)$ & $28,853(1.8 \%)$ & $3162(2.8 \%)$ & $<0.0001$ \\
\hline Mild liver disease & $8437(0.49 \%)$ & $7918(0.49 \%)$ & $519(0.46 \%)$ & 0.17 \\
\hline Diabetes & $315,236(18.4 \%)$ & $289,481(18.1 \%)$ & $25,755(23.1 \%)$ & $<0.0001$ \\
\hline Diabetes with end organ damage & $92,786(5.4 \%)$ & $84,100(5.3 \%)$ & $8686(7.8 \%)$ & $<0.0001$ \\
\hline Hemiplegia & $13,668(0.80 \%)$ & 11,972 (0.75\%) & 1696 (1.5\%) & $<0.0001$ \\
\hline Renal failure/disease & $58,438(3.4 \%)$ & $53,802(3.4 \%)$ & $4636(4.2 \%)$ & $<0.0001$ \\
\hline Any tumor, leukemia, or lymphoma & $172,705(10.1 \%)$ & $160,676(10.0 \%)$ & $12,029(10.8 \%)$ & $<0.0001$ \\
\hline Moderate or severe liver disease & $1969(0.11 \%)$ & $1852(0.12 \%)$ & $117(0.10 \%)$ & 0.30 \\
\hline Metastatic cancer & $17,879(1.0 \%)$ & $17,113(1.1 \%)$ & $766(0.69 \%)$ & $<0.0001$ \\
\hline AIDS & $546(0.03 \%)$ & 519 (0.03\%) & $27(0.02 \%)$ & 0.14 \\
\hline Hypertension & $823,180(48.1 \%)$ & 754,449 (47.1\%) & $68,731(61.6 \%)$ & $<0.0001$ \\
\hline Hyperlipidemia & $597,299(34.9 \%)$ & $554,334(34.6 \%)$ & 42,965 (38.5\%) & $<0.0001$ \\
\hline Coronary artery disease & $299,331(17.5 \%)$ & 273,719 (17.1\%) & $25,612(22.9 \%)$ & $<0.0001$ \\
\hline
\end{tabular}

SD standard deviation, N/A not applicable

Bold represents statistical significance, with a $P$-value $<0.05$

ancident dementia defined as the occurrence of two claims at least four-weeks apart with ICD-9-CM codes, 290.xx, 294.1x, or 331.2, with baseline 365 days without any claim for dementia

${ }^{\mathrm{b}}$ Met eligibility criteria and did not have dementia in the baseline 365-day period

hyperuricemia may be a compensatory mechanism to counteract oxidative damage related to atherosclerosis and aging in humans [38]. However, most evidence points to hyperuricemia being a risk factor for cardiovascular disease [39-42]. It is possible that the association of hyperuricemia with cardiovascular disease is due to concomitant oxidative stress (a hypothesis that remains to be proven), which is implicated in the pathogenesis of 
Table 2 Association of gout and other potential risk factors with incident dementia in adults 65 years or older

\begin{tabular}{|c|c|c|c|c|c|c|}
\hline & \multicolumn{2}{|c|}{ Multivariable-adjusted (Model 1) } & \multicolumn{2}{|c|}{$\begin{array}{l}\text { Multivariable-adjusted } \\
(\text { Model 2) }\end{array}$} & \multicolumn{2}{|c|}{$\begin{array}{l}\text { Multivariable-adjusted } \\
\text { (Model 3) }^{\mathrm{a}}\end{array}$} \\
\hline & $\mathrm{HR}(95 \% \mathrm{Cl})$ & $P$-value & $\mathrm{HR}(95 \% \mathrm{Cl})$ & $P$-value & $\mathrm{HR}(95 \% \mathrm{Cl})$ & $P$-value \\
\hline \multicolumn{7}{|l|}{ Age (in years) } \\
\hline $65-<75$ & Ref & & Ref & & Ref & \\
\hline $75-<85$ & $3.53(3.48,3.58)$ & $<0.0001$ & $3.49(3.44,3.54)$ & $<0.0001$ & $3.35(3.30,3.40)$ & $<0.0001$ \\
\hline$\geq 85$ & $7.81(7.68,7.95)$ & $<0.0001$ & $7.75(7.62,7.88)$ & $<0.0001$ & $6.80(6.68,6.92)$ & $<0.0001$ \\
\hline \multicolumn{7}{|l|}{ Sex } \\
\hline Male & Ref & & Ref & & Ref & \\
\hline Female & $1.34(1.32,1.36)$ & $<0.0001$ & $1.33(1.31,1.34)$ & $<0.0001$ & $1.26(1.25,1.28)$ & $<0.0001$ \\
\hline \multicolumn{7}{|l|}{ Race } \\
\hline White & Ref & & Ref & & Ref & \\
\hline Black & $1.29(1.26,1.32)$ & $<0.0001$ & $1.33(1.30,1.35)$ & $<0.0001$ & $1.24(1.22,1.27)$ & $<0.0001$ \\
\hline Other & $0.87(0.85,0.89)$ & $<0.0001$ & $0.89(0.87,0.92)$ & $<0.0001$ & $0.85(0.82,0.87)$ & $<0.0001$ \\
\hline $\begin{array}{l}\text { Charlson-Romano score, } \\
\text { per unit change }\end{array}$ & $1.16(1.15,1.16)$ & $<0.0001$ & N/A & & N/A & \\
\hline \multicolumn{7}{|l|}{ Charlson-Romano score } \\
\hline 0 & N/A & & Ref & & N/A & \\
\hline 1 & & & $1.72(1.68,1.75)$ & $<0.0001$ & & \\
\hline$\geq 2$ & & & $2.17(2.14,2.19)$ & $<0.0001$ & & \\
\hline Gout & $1.15(1.12,1.18)$ & $<0.0001$ & $1.17(1.14,1.20)$ & $<0.0001$ & $1.17(1.14,1.21)$ & $<0.0001$ \\
\hline
\end{tabular}

N/A not applicable, $H R$ hazard ratio, $C l$ confidence interval, Ref referent category

Bold represents statistical significance, with a $P$-value $<0.05$

${ }^{a}$ Model 1 included Charlson-Romano score as a continuous variable; Model 2 replaced it with categorized Charlson-Romano score; and Model 3 replaced it with each of the 17 Charlson-Romano comorbidities plus hypertension, hyperlipidemia, and coronary artery disease. All models were also adjusted for medications for cardiovascular diseases (statins, beta-blockers, diuretics, ACE-inhibitors) and for urate-lowering therapies for gout (allopurinol, febuxostat)

dementia [32, 43, 44], or due to its association with other cardiovascular disease risk factors [45].

We found that gout was associated with a significantly increased risk of dementia in people without each of the common comorbidities, CAD, hyperlipidemia, CVD, diabetes, or hypertension. On the other hand, gout was not associated with dementia risk in in the presence of any of these comorbidities. This is an interesting finding from the subgroup analyses and novel, to our knowledge. Whether this indicates that the key pathways of disease causation in dementia differ based on the presence/absence of these conditions or that gout only imparts the dementia risk in the absence of these conditions, remains to be seen. This finding needs to be reproduced in other elderly cohorts.

Our study has several limitations that must be considered while interpreting findings. Our findings are from a representative elderly population, 65 years or older, and therefore, findings should not be generalized to younger populations. Several limitations are related to claims data we used. Despite the use of a valid diagnostic code algorithm to identify people with dementia, our study findings are at the risk of non-differential misclassification bias, since the measurement error in dementia diagnosis (study outcome) is unlikely to have differed by the exposure (gout) status. This may have biased the results towards the null, making these results conservative estimates. Dementia diagnosis was not based on diagnostic criteria, MMSE scores, or magnetic resonance imaging (MRI) characteristics, since these data are not available in Medicare claims. Availability of such data could have allowed additional insights into the observed association and possibly reduced the misclassification bias. Due to an observational study design, residual confounding is possible; we included several potential confounders and covariates to reduce the confounding bias. A delay or failure of diagnosis of dementia may have occured if people with symptoms of dementia were late or fail to seek medical help. A surveillance bias in people diagnosed with gout for other chronic conditions such as dementia may have led to a higher rate of the diagnosis of dementia to some extent. However, there are no previous known associations of gout with dementia and on the contrary some studies 

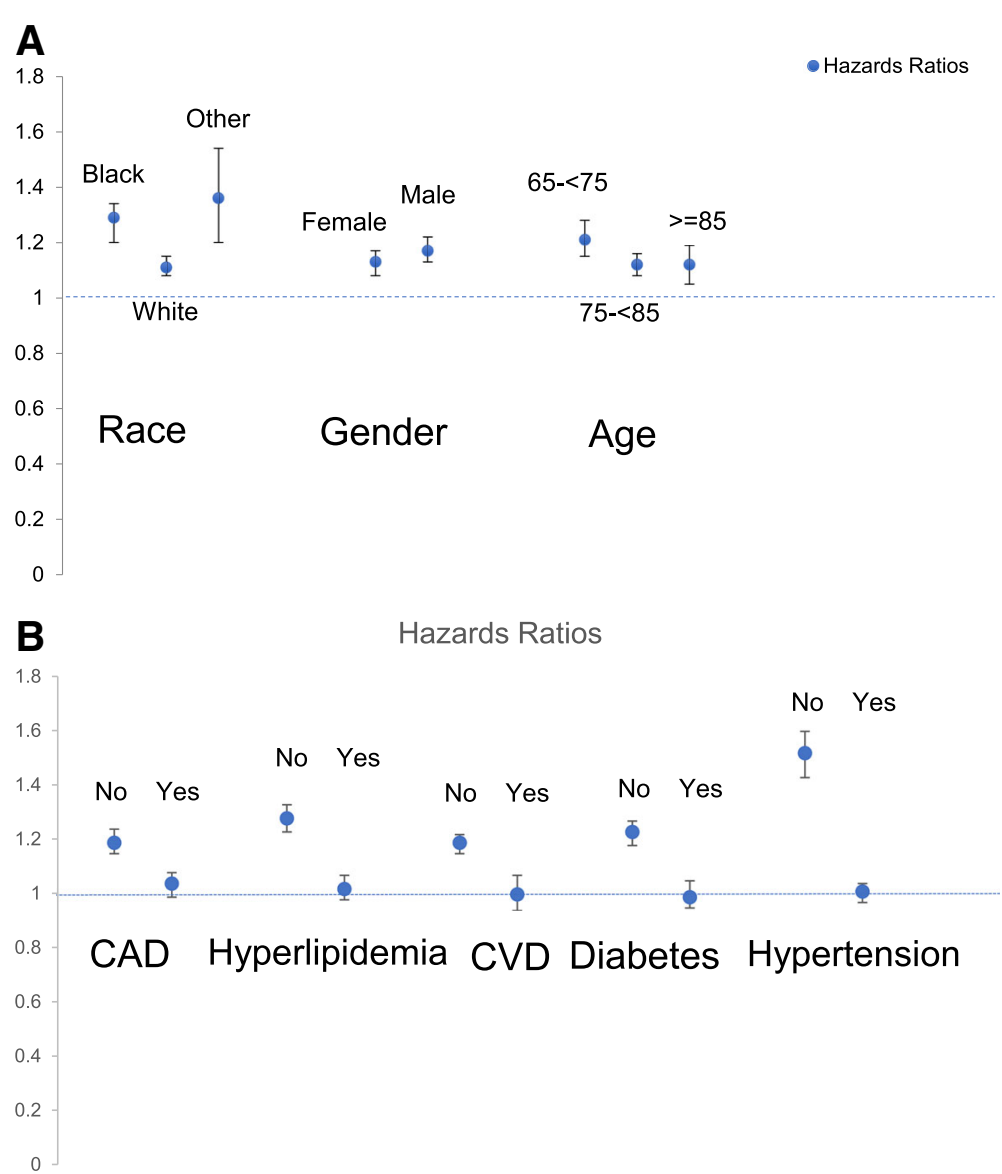

Fig. 1 Association of gout with incident dementia by subgroups- Race, sex and age (a); presence/absence of CAD, hyperlipidemia, CVD, diabetes and hypertension (b). Point estimates are indicated by filled circles and the whiskers represent the $95 \%$ confidence intervals. Hazards ratio are statistically non-significant when the confidence interval includes the null hazard ratio of 1.0. For example, none of the associations of gout with incident dementia were significant in the presence of key comorbidities (CAD, hyperlipidemia, CVD, diabetes and hypertension) except borderline significance in the presence of CAD. P-values for interaction terms were as follows: Gout*age, $p<0.0001$; Sex*gout, $p=0.0018$; Gout*race, $p=0.035$; Gout ${ }^{*}$ CAD $p<0.0001$; Gout*hyperlipidemia $p<0.0001$; Gout ${ }^{*}$ CVD $p<0.0001$; Gout*diabetes $p<0.0001$; Gout*hypertension $p<0.0001$

previously showed hyperuricemia (a cardinal feature of gout) to be associated with a lower risk of dementia [22, 23], making this surveillance bias unlikely. Medicare data does not include body mass index data, which limited our ability to account for the role that obesity may play in this relationship, or whether general versus abdominal obesity differs in their effect on the noted association. The lack of the availability of laboratory data in the Medicare claims limited our ability to further investigate whether the noted association was mediated by hyperuricemia, chronic inflammation and/or oxidative stress associated with gout. This question needs further study with different study design and data source. A study of dementia subtypes was outside the scope of this study, and needs to be addressed with future studies. Study strengths included a large sample size, robustness of findings in sensitivity analyses and use of a U.S. representative population of the elderly.

\section{Conclusions}

In conclusion, we found that gout was independently associated with a $15-18 \%$ higher hazard of dementia in the elderly. This increased risk might be due to the effects of hyperuricemia, oxidative stress, and/or inflammation. Future studies should also explore whether interventions targeting these can ameliorate this increased risk.

\section{Abbreviations}

ACE inhibitor: Angiotensin converting enzyme inhibitor; CMS: Centers for Medicare and Medicaid Services; ICD-9-CM: International Classification of Diseases, ninth revision, common modification; IRB: Institutional Review Board; STROBE: Strengthening of Reporting in Observational studies in Epidemiology; UAB: University of Alabama at Birmingham 


\section{Acknowledgements}

We thank Dr. Jeffrey Curtis of the UAB Division of Rheumatology, who permitted us to re-use the $5 \%$ Medicare data. We thank patients at the University of Alabama gout clinic for asking us questions about comorbidities of gout and whether gout may be related to their other conditions, which prompted us to ask this question. These data were presented as a poster presentation at the 2018 American College of Rheumatology (ACR) Annual meeting at Chicago, IL, USA. Therefore, some of the text of the study abstract was included in and published as part of our presented abstract at this meeting.

\section{Funding}

This material is the result of work supported by research funds from the Division of Rheumatology at the University of Alabama at Birmingham and the resources and use of facilities at the Birmingham VA Medical Center, Birmingham, Alabama, USA. The funding body did not play any role in design, in the collection, analysis, and interpretation of data; in the writing of the manuscript; and in the decision to submit the manuscript for publication.

\section{Availability of data and materials}

The provider of the data, Centers for Medicaid and Medicare (CMS) data warehouse, does not allow us to publicly deposit datasets according to the data use agreement, in accordance with patient privacy and confidentiality rules. We will make data available to colleagues, after appropriate approvals from the Institutional Review Boards and Centers for Medicare and Medicaid services (CMS) data warehouse have been obtained.

\section{Authors' contributions}

JAS developed study concept and design, study protocol, obtained funding and provided administrative, technical or material support reviewed and interpreted all results and drafted the manuscript. JDC acquired and programmed all data, performed statistical analyses, interpreted results and performed critical revision of the manuscript for important intellectual content. Both authors read and approved the final manuscript.

\section{Ethics approval and consent to participate}

The University of Alabama at Birmingham's Institutional Review Board approved this study and waived the need for informed consent for this database study. All investigations were conducted in conformity with ethical principles of research.

\section{Consent for publication}

Not applicable

\section{Competing interests}

JAS has received consultant fees from Crealta/Horizon, Fidia, UBM LLC, Medscape, WebMD, the National Institutes of Health and the American College of Rheumatology. JAS owns stock options in Amarin pharmaceuticals, Inc. JAS is a member of the Veterans Affairs Rheumatology Field Advisory Committee. JAS is the editor and the Director of the UAB Cochrane Musculoskeletal Group Satellite Center on Network Meta-analysis. JAS served as a member of the American College of Rheumatology's (ACR) Annual Meeting Planning Committee (AMPC) and Quality of Care Committees, the Chair of the ACR Meet-the-Professor, Workshop and Study Group Subcommittee and the co-chair of the ACR Criteria and Response Criteria subcommittee. JAS is a member of the executive of OMERACT, an organization that develops outcome measures in rheumatology and receives arms-length funding from 36 companies. DC has no conflicts to declare. There are no non-financial competing interests for any of the authors.

\section{Publisher's Note}

Springer Nature remains neutral with regard to jurisdictional claims in published maps and institutional affiliations.

\section{Author details}

${ }^{1}$ Medicine Service, Birmingham VA Medical Center 700 19th St S, Birmingham, AL 35233, USA. ${ }^{2}$ Department of Medicine at School of Medicine, University of Alabama at Birmingham, 1720 Second Ave. South, Birmingham, AL 35294-0022, USA. ${ }^{3}$ Division of Epidemiology at School of Public Health, University of Alabama at Birmingham, 1720 Second Ave.
South, Birmingham, AL 35294-0022, USA. ${ }^{4}$ University of Alabama at Birmingham, Faculty Office Tower 805B, 510 20th Street S, Birmingham, AL 35294-0022, USA.

Received: 15 March 2018 Accepted: 6 November 2018

Published online: 14 November 2018

\section{References}

1. Dementia and Alzheimer's leading cause of death in England and Wales. https://www.theguardian.com/society/2016/nov/14/dementia-andalzheimers-leading-cause-of-death-england-and-wales. Accessed $10 \mathrm{Nov}$ 2018.

2. Dubois B, Picard G, Sarazin M. Early detection of Alzheimer's disease: new diagnostic criteria. Dialogues Clin Neurosci. 2009;11(2):135-9.

3. Tombaugh TN, McIntyre NJ. The mini-mental state examination: a comprehensive review. J Am Geriatr Soc. 1992;40(9):922-35.

4. Reisberg B, Ferris SH, de Leon MJ, Crook T. The global deterioration scale for assessment of primary degenerative dementia. Am J Psychiatry. 1982;139(9):1136-9.

5. Feldman HH, Jacova C, Robillard A, Garcia A, Chow T, Borrie M, Schipper HM, Blair M, Kertesz A, Chertkow H. Diagnosis and treatment of dementia: 2. Diagnosis. CMAJ. 2008;178(7):825-36.

6. Erkinjuntti T. Clinical criteria for vascular dementia: the NINDS-AIREN criteria. Dementia. 1994:5(3-4):189-92.

7. Dubois B, Feldman HH, Jacova C, Dekosky ST, Barberger-Gateau P, Cummings J, Delacourte A, Galasko D, Gauthier S, Jicha G, et al. Research criteria for the diagnosis of Alzheimer's disease: revising the NINCDS-ADRDA criteria. Lancet Neurol. 2007;6(8):734-46.

8. Humpel C, Hochstrasser T. Cerebrospinal fluid and blood biomarkers in Alzheimer's disease. World J Psychiatry. 2011;1(1):8-18.

9. Humpel C. Identifying and validating biomarkers for Alzheimer's disease. Trends Biotechnol. 2011;29(1):26-32.

10. Aguero-Torres H, Fratiglioni L, Guo Z, Viitanen M, von Strauss E, Winblad B. Dementia is the major cause of functional dependence in the elderly: 3 year follow-up data from a population-based study. Am J Public Health. 1998;88(10):1452-6.

11. Banerjee S, Smith SC, Lamping DL, Harwood RH, Foley B, Smith P, Murray J, Prince $M$, Levin E, Mann A, et al. Quality of life in dementia: more than just cognition. An analysis of associations with quality of life in dementia. J Neurol Neurosurg Psychiatry. 2006;77(2):146-8.

12. Bunn F, Burn AM, Goodman C, Rait G, Norton S, Robinson L, Schoeman J, Brayne C. Comorbidity and dementia: a scoping review of the literature. BMC Med. 2014;12:192.

13. Johnson NB, Hayes LD, Brown K, Hoo EC, Ethier KA, Centers for Disease C, Prevention. CDC National Health Report: leading causes of morbidity and mortality and associated behavioral risk and protective factors--United States, 2005-2013. MMWR Suppl. 2014;63(4):3-27.

14. Prince $M$, Bryce $R$, Albanese E, Wimo A, Ribeiro W, Ferri CP. The global prevalence of dementia: a systematic review and metaanalysis. Alzheimers Dement. 2013;9(1):63-75 e62.

15. Launer LJ, Andersen K, Dewey ME, Letenneur L, Ott A, Amaducci LA, Brayne C, Copeland JR, Dartigues JF, Kragh-Sorensen P, et al. Rates and risk factors for dementia and Alzheimer's disease: results from EURODEM pooled analyses. EURODEM incidence research group and work groups. European studies of dementia. Neurology. 1999;52(1):78-84.

16. Afsar B, Elsurer R, Covic A, Johnson RJ, Kanbay M. Relationship between uric acid and subtle cognitive dysfunction in chronic kidney disease. Am J Nephrol. 2011;34(1):49-54.

17. Cicero AF, Desideri G, Grossi G, Urso R, Rosticci M, D'Addato S, Borghi C Brisighella Heart Study G. Serum uric acid and impaired cognitive function in a cohort of healthy young elderly: data from the Brisighella study. Intern Emerg Med. 2015;10(1):25-31.

18. Perna $\mathrm{L}$, Mons $\mathrm{U}$, Schottker B, Brenner $\mathrm{H}$. Association of cognitive function and serum uric acid: are cardiovascular diseases a mediator among women? Exp Gerontol. 2016;81:37-41.

19. Verhaaren BF, Vernooij MW, Dehghan A, Vrooman HA, de Boer R, Hofman A, Witteman JC, Niessen WJ, Breteler MM, van der Lugt A, et al. The relation of uric acid to brain atrophy and cognition: the Rotterdam scan study. Neuroepidemiology. 2013;41(1):29-34

20. Beydoun MA, Canas JA, Dore GA, Beydoun HA, Rostant OS, FanelliKuczmarski MT, Evans MK, Zonderman AB. Serum uric acid and its 
association with longitudinal cognitive change among urban adults. J Alzheimers Dis. 2016;52(4):1415-30.

21. Ruggiero C, Cherubini A, Lauretani F, Bandinelli S, Maggio M, Di lorio A, Zuliani G, Dragonas C, Senin U, Ferrucci L. Uric acid and dementia in communitydwelling older persons. Dement Geriatr Cogn Disord. 2009;27(4):382-9.

22. Euser SM, Hofman A, Westendorp RG, Breteler MM. Serum uric acid and cognitive function and dementia. Brain. 2009;132(Pt 2):377-82.

23. Hong JY, Lan TY, Tang GJ, Tang CH, Chen TJ, Lin HY. Gout and the risk of dementia: a nationwide population-based cohort study. Arthritis Res Ther. 2015:17:139.

24. Latourte A, Soumare A, Bardin T, Perez-Ruiz F, Debette S, Richette P. Uric acid and incident dementia over 12 years of follow-up: a population-based cohort study. Ann Rheum Dis. 2018;77:328-35.

25. Singh JA, Hodges JS, Toscano JP, Asch SM. Quality of care for gout in the US needs improvement. Arthritis Rheum. 2007:57(5):822-9.

26. Romano PS, Roos LL, Jollis JG. Adapting a clinical comorbidity index for use with ICD-9-CM administrative data: differing perspectives. J Clin Epidemiol. 1993;46(10):1075-9 discussion 1081-1090.

27. Quan H, Li B, Saunders LD, Parsons GA, Nilsson Cl, Alibhai A, Ghali WA Investigators I. Assessing validity of ICD-9-CM and ICD-10 administrative data in recording clinical conditions in a unique dually coded database. Health Serv Res. 2008;43(4):1424-41.

28. U.S. Census Bureau. National Population Projections. I. Summary files. Total population by age, sex, race and Hispanic origin. (NP-T4-F). Projections of the total resident population by 5-year age groups, race and Hispanic origin with special age categories: middle series. 2025 to 2045. Available at: https:/wwww2.census.gov/programs-surveys/popproj/tables/2000/2000national-summary-tables/np-t4-f.pdf. Accessed 10 Nov 2018.

29. Banta JE. Dementia: morbidity and medications. Age Ageing. 2017;46(1):4-5.

30. Sanderson M, Wang J, Davis DR, Lane MJ, Cornman CB, Fadden MK. Comorbidity associated with dementia. Am J Alzheimers Dis Other Demen. 2002:17(2):73-8.

31. Witthaus E, Ott A, Barendregt JJ, Breteler M, Bonneux L. Burden of mortality and morbidity from dementia. Alzheimer Dis Assoc Disord. 1999;13(3):176-81.

32. Lin MT, Beal MF. Mitochondrial dysfunction and oxidative stress in neurodegenerative diseases. Nature. 2006:443(7113):787-95.

33. Pratico D, Uryu K, Leight S, Trojanoswki JQ, Lee VM. Increased lipid peroxidation precedes amyloid plaque formation in an animal model of Alzheimer amyloidosis. J Neurosci. 2001;21 (12):4183-7.

34. Bagnati M, Perugini C, Cau C, Bordone R, Albano E, Bellomo G. When and why a water-soluble antioxidant becomes pro-oxidant during copperinduced low-density lipoprotein oxidation: a study using uric acid. Biochem J. 1999;340(Pt 1):143-52.

35. Kanellis J, Kang DH. Uric acid as a mediator of endothelial dysfunction, inflammation, and vascular disease. Semin Nephrol. 2005;25(1):39-42.

36. Squadrito GL, Cueto R, Splenser AE, Valavanidis A, Zhang H, Uppu RM, Pryor WA. Reaction of uric acid with peroxynitrite and implications for the mechanism of neuroprotection by uric acid. Arch Biochem Biophys. 2000;376(2):333-7.

37. Yu ZF, Bruce-Keller AJ, Goodman Y, Mattson MP. Uric acid protects neurons against excitotoxic and metabolic insults in cell culture, and against focal ischemic brain injury in vivo. J Neurosci Res. 1998;53(5):613-25.

38. Nieto FJ, Iribarren C, Gross MD, Comstock GW, Cutler RG. Uric acid and serum antioxidant capacity: a reaction to atherosclerosis? Atherosclerosis. 2000;148(1):131-9.

39. Feig DI, Kang DH, Johnson RJ. Uric acid and cardiovascular risk. N Engl J Med. 2008;359(17):1811-21.

40. Kim SC, Liu J, Solomon DH. Risk of incident atrial fibrillation in gout: a cohort study. Ann Rheum Dis. 2016;75(8):1473-8.

41. Kim SY, Guevara JP, Kim KM, Choi HK, Heitjan DF, Albert DA. Hyperuricemia and risk of stroke: a systematic review and meta-analysis. Arthritis Rheum. 2009:61(7):885-92.

42. Kim SY, Guevara JP, Kim KM, Choi HK, Heitjan DF, Albert DA. Hyperuricemia and coronary heart disease: a systematic review and meta-analysis. Arthritis Care Res. 2010;62(2):170-80.

43. Coyle JT, Puttfarcken P. Oxidative stress, glutamate, and neurodegenerative disorders. Science. 1993;262(5134):689-95.

44. Barnham KJ, Masters CL, Bush Al. Neurodegenerative diseases and oxidative stress. Nat Rev Drug Discov. 2004;3(3):205-14.

45. Culleton BF, Larson MG, Kannel WB, Levy D. Serum uric acid and risk for cardiovascular disease and death: the Framingham heart study. Ann Intern Med. 1999;131(1):7-13.

Ready to submit your research? Choose BMC and benefit from:

- fast, convenient online submission

- thorough peer review by experienced researchers in your field

- rapid publication on acceptance

- support for research data, including large and complex data types

- gold Open Access which fosters wider collaboration and increased citations

- maximum visibility for your research: over $100 \mathrm{M}$ website views per year

At BMC, research is always in progress.

Learn more biomedcentral.com/submissions 